\title{
Recommandations canadiennes provisoires concernant l'utilisation d'une dose fractionnée du vaccin contre la fièvre jaune durant une pénurie du vaccin
}

Groupe de travail sur la fièvre jaune ${ }^{1}$, au nom du Comité consultatif de la médecine tropicale et de la médecine des voyages (CCMTMV)

\section{Résumé}

Cette déclaration décrit les recommandations provisoires destinées à être utilisées uniquement durant les pénuries de vaccin contre la fièvre jaune. Les recommandations sont différentes des recommandations standard relatives à la vaccination contre la fièvre jaune dans le Guide canadien d'immunisation et dans la déclaration sur la fièvre jaune à l'intention des voyageurs du CCMTMV.

\author{
Affiliation \\ ${ }^{1}$ Remarque : Les membres du \\ Groupe de travail sur la fièvre \\ jaune sont énumérés dans la \\ section Remerciements
}

Correspondance : catmat. secretariat@phac-aspc.gc.ca

Citation proposée : Groupe de travail sur la fièvre jaune, au nom du Comité consultatif de la médecine tropicale et de la médecine des voyages (CCMTMV). Recommandations canadiennes provisoires concernant l'utilisation d'une dose fractionnée du vaccin contre la fièvre jaune durant une pénurie du vaccin. Relevé des maladies transmissibles au Canada 2016;42:177-9. https://doi.org/10.14745/ccdr.v42i08a02f

\section{Introduction}

Les pénuries de vaccin contre la fièvre jaune posent un défi. Les cliniques de santé-voyage ne recevront qu'une petite fraction du nombre de vaccins habituellement commandés; il se pourrait même que certaines cliniques de voyage n'aient accès à aucun vaccin contre la fièvre jaune jusqu'au prochain approvisionnement disponible du vaccin. II n'existe à l'heure actuelle qu'un seul fabricant homologué du vaccin au Canada.

En 2016, il y a eu des demandes pour l'utilisation d'une fraction de la dose du vaccin contre la fièvre jaune pour combler une pénurie globale du vaccin, une mesure qui permettrait de vacciner un plus grand nombre de personnes durant la pénurie de vaccins (1-3). Cette suggestion se fonde principalement sur trois études ayant démontré que les doses de l'ordre du dixième $(1 / 10)$ au cinquième $(1 / 5)$ de la dose habituelle de $0,5 \mathrm{~mL}$ par voie sous-cutanée conféreraient une protection contre le virus selon les critères de laboratoire.

Le 17 juin 2016, l'Organisation mondiale de la Santé (OMS) a publié une déclaration indiquant que le Groupe stratégique consultatif d'experts (SAGE) sur la vaccination de l'OMS avait découvert que I'utilisation du cinquième de la dose standard du vaccin (à savoir $0,1 \mathrm{~mL}$ plutôt que $0,5 \mathrm{~mL}$ ) peut offrir une protection contre la fièvre jaune pendant une période d'au moins 12 mois selon l'examen des données existantes (4). L'OMS déclare que cette dose fractionnée du vaccin contre la fièvre jaune peut être considérée comme une mesure sécuritaire et efficace de contrôle des éclosions en milieu urbain en cas de pénurie de vaccins.
Le CCMTMV a mis sur pied un groupe de travail pour examiner les données probantes et formuler des recommandations provisoires concernant l'utilisation et la consignation des doses fractionnées du vaccin contre la fièvre jaune au Canada qui sont destinées à être utilisées uniquement durant les pénuries de ce vaccin. Chaque membre était bénévole, et aucun d'entre eux n'a signalé de conflit d'intérêts pertinent. Les recommandations sont différentes des recommandations standard relatives à la vaccination contre la fièvre jaune dans le Guide canadien d'immunisation (5) et dans la déclaration sur la fièvre jaune à l'intention des voyageurs du CCMTMV (6).

\section{Méthodologie}

Une recherche documentaire a été réalisée afin de trouver des données liées à l'immunogénicité des doses fractionnées du vaccin contre la fièvre jaune. Les recherches effectuées dans les bases de données électroniques Ovid Medline, Embase, Global Health et Scopus ont permis d'extraire diverses données probantes. La recherche s'est échelonnée de la date initiale respective de chaque base de données à juin 2016 et 49 résultats ont été conservés. Les titres et les résumés de ces résultats ont été examinés puis sélectionnés aux fins $d$ 'inclusion en fonction de la pertinence de la question de recherche.

\section{Résultats}

En 2008, Roukens et ses collaborateurs ont étudié l'effet d'une dose cinq fois plus faible que celle du vaccin contre la fièvre 
jaune administrée par voie intradermique. Tous les sujets ont présenté des titres d'anticorps neutralisants considérés comme protecteurs (7). L'âge des sujets adultes variait considérablement (de 18 à 70 ans), la moyenne s'établissant à 27 ans.

En 2013, Martins et ses collaborateurs ont étudié la séroconversion et les réponses à la virémie après I'utilisation d'une dose complète et de cinq dilutions différentes de la dose humaine habituelle du vaccin contre la fièvre jaune 17-DD administré par voie sous-cutanée (8). Les différences observées dans les réponses immunitaires ont été faibles jusqu'à une dilution de 1:50.

Au cours de la phase de prolongation de l'étude de Martins, réalisée en 2014 et utilisant les mêmes données et prélèvements sanguins des patients, Campi-Azevedo a étudié les biomarqueurs sériques des réponses immunitaires à médiation cellulaire au moyen de sous-doses (9). Les données ont montré la présence d'une protection jusqu'à des dilutions de 1:50. En revanche, les résultats de l'ensemble des marqueurs de l'immunité (sérologie, virémie et immunité à médiation cellulaire) n'ont été systématiquement équivalents à ceux de la dose complète qu'à des dilutions allant jusqu'à 1:10. Les évaluations de Martins et de Campi-Azevedo portaient toutes sur de jeunes hommes en santé âgés en moyenne de 19 ans.

Bien que les résultats de ces études soient encourageants, ces données demeurent limitées. Des recherches plus approfondies sont nécessaires afin de déterminer l'efficacité des doses fractionnées, surtout chez les jeunes enfants.

\section{Recommandations}

Dans des circonstances normales, la recommandation d'utiliser une dose fractionnée du vaccin contre la fièvre jaune ne serait pas émise pour les voyageurs. Toutefois, il se peut que certains voyageurs se rendant dans des régions où la fièvre jaune est endémique ou épidémique n'aient pas accès à une dose complète du vaccin contre la fièvre jaune. Par conséquent, ces derniers n'ont d'autre choix que de ne recevoir aucun vaccin ou une dose fractionnée du vaccin.

Compte tenu de cette situation, le CCMTMV émet les recommandations ci-dessous, applicables aux personnes pour qui les recommandations standard relatives au vaccin contre la fièvre jaune s'appliquent, y compris les jeunes enfants :

- Pour les voyages dans une région d'un pays présentant un risque de fièvre jaune, et tout particulièrement celles aux prises avec une éclosion en cours, les professionnels de la santé doivent d'abord mettre l'accent sur l'importance de recevoir une dose complète du vaccin ou autrement de remettre à plus tard le voyage.

- Si un voyageur doit voyager dans une région endémique, en particulier une région touchée par une éclosion de fièvre jaune, et qu'une dose complète du vaccin ne peut lui être offerte après le déploiement d'efforts raisonnables pour trouver une telle dose, une dose fractionnée peut lui être administrée. La dose doit être cing fois plus faible que la dose habituelle $(0,1 \mathrm{~mL}$ plutôt que $0,5 \mathrm{~mL})$ et doit être administrée par la voie classique sous-cutanée. À l'instar de la dose complète, l'effet protecteur de la dose fractionnée commence 10 jours après son administration à une personne n'ayant jamais reçu le vaccin contre la fièvre jaune.

- $\quad$ Si un voyageur qui prévoit un itinéraire à risque élevé reçoit une dose fractionnée du vaccin contre la fièvre jaune, et plus tard, apprend qu'une dose normale est offerte, cette dose peut être administrée et le Certificat international de vaccination ou de prophylaxie peut être délivré.

- Une fois le flacon du vaccin reconstitué, ce dernier doit être conservé à une température comprise entre 2 et $8^{\circ} \mathrm{C}$ et être utilisé dans l'heure qui suit, ce qui implique qu'il sera nécessaire de vacciner plusieurs personnes au cours de cette même heure afin d'utiliser efficacement le contenu du flacon à l'intérieur du temps alloué. Une façon de gaspiller moins de vaccin pour le professionnel de la santé est d'utiliser les seringues d'insuline de $1 \mathrm{cc}$ jetables munies d'une aiguille non détachable. Un flacon peut fournir jusqu'à quatre, voire cinq, doses. Observer les règles rigoureuses d'asepsie.

- Si moins de cinq doses sont prévues, on recommande d'utiliser l'ensemble du contenu du flacon et de le répartir de façon égale aux personnes présentes pour la vaccination, ce qui permettra d'administrer une dose un peu plus élevée que $0,1 \mathrm{~mL}$ à chaque personne.

- Selon les données disponibles, une dose fractionnée $(1 / 5)$ peut être considérée comme protectrice pendant une période d'un an. La protection pourrait être plus longue, bien qu'on ne dispose pas de données à long terme. Aucune recommandation n'a été formulée à ce jour sur l'administration répétée de doses fractionnées pour des voyages subséquents.

- Dès que l'approvisionnement du vaccin contre la fièvre jaune sera rétabli, l'usage des doses fractionnées devrait cesser.

- On rappelle aux professionnels de la santé que l'OMS considère maintenant que l'administration d'une seule dose complète du vaccin contre la fièvre jaune offre une protection à vie, peu importe la date à laquelle elle est administrée.

\section{Documentation de la vaccination contre la fièvre jaune au moyen d'une dose fractionnée}

L'OMS souligne que les doses fractionnées du vaccin contre la fièvre jaune ne sont pas admissibles aux certificats de vaccination contre la fièvre jaune en vertu du Règlement sanitaire international (4). Par conséquent, le CCMTMV ne recommande pas aux praticiens d'utiliser la carte officielle du Certificat international de vaccination ou de prophylaxie (CIVP) pour documenter l'administration d'une dose fractionnée.

On suggère à la place d'utiliser le Certificat de contre-indication médicale à la vaccination fourni par l'Agence de la santé publique du Canada. Une explication peut être rédigée à 
I'intérieur de ce dernier informant qu'une dose fractionnée de $0,1 \mathrm{~mL}$ du vaccin contre la fièvre jaune par voie sous-cutanée a été administrée en raison d'une grave pénurie de vaccins.

\section{Autres ressources et liens utiles}

Gouvernement du Canada - Centres de vaccination contre la fièvre jaune au Canada. http://www.phac-aspc.gc.ca/tmp-pmv/ yf-fj/index-fra.php

Organisation mondiale de la Santé - Exigences et recommandations à l'égard de la vaccination des voyageurs internationaux. http://www.who.int/ith/fr/

\section{Remerciements}

La présente déclaration a été rédigée par le groupe de travail sur la fièvre jaune composé des personnes suivantes : Teitelbaum P (président), Bui Y, Libman M, Pernica J et Abdel-Motagally M.

Membres du CCMTMV : McCarthy A (présidente), Acharya A, Boggild A, Brophy J, Bui Y, Crockett M, Greenaway C, Libman M, Teitelbaum $P$ et Vaughan $S$.

Membres du groupe de liaison : Audcent $T$ (Société canadienne de pédiatrie), Gershman M (Centers for Disease Control and Prevention des États-Unis) et Pernica J (Association pour la microbiologie médicale et l'infectiologie Canada).

Membres d'office : Marion D (Centre des services de santé des Forces canadiennes, ministère de la Défense nationale), Rossi C (Direction du groupe des services de santé des Forces canadiennes, ministère de la Défense nationale), McDonald $\mathrm{P}$ (Division des médicaments anti-infectieux, Santé Canada) et Schofield S (Entomologie de la lutte antiparasitaire, ministère de la Défense nationale).

\section{Conflit d'intérêts}

Aucun déclaré.

\section{Références}

1. Monath TP, Vasconcelos PF. Yellow fever. J Clin Virol 2015 Mar;64:160-173.

2. Monath TP, Woodall JP, Gubler DJ, Yuill TM, Mackenzie JS, Martins RM, et al. Yellow fever vaccine supply: a possible solution. Lancet 2016 Apr 16;387(10028):1599-1600.

3. Lucey D, Gostin LO. A Yellow Fever Epidemic: A New Global Health Emergency? JAMA 2016 May 9.

4. Organisation mondiale de la Santé. Utilisation de doses réduites de vaccin antiamaril dans les situations d'urgences. 2016 http://www.who.int/mediacentre/news/ statements/2016/yellow-fever-vaccine/fr/.

5. Agence de la santé publique du Canada. Guide canadien d'immunisation. Partie 4 : Vaccins actifs, Vaccin contre la fièvre jaune. 2012 http:///publicat/cig-gci/p04-yfev-fiej-fra. php.

6. Comité consultatif de la médecine tropicale et de la médecine des voyages (CCMTMV). Déclaration sur la fièvre jaune à l'intention des voyageurs. Relevé des maladies transmissibles au Canada 2013;39(ACS-2). http://www.phacaspc.gc.ca/publicat/ccdr-rmtc/13vol39/acs-dcc-2/index-fra. php.

7. Roukens AH, Vossen AC, Bredenbeek PJ, van Dissel JT, Visser LG. Intradermally administered yellow fever vaccine at reduced dose induces a protective immune response: a randomized controlled non-inferiority trial. PLoS ONE 2008;3(4):e1993.

8. Martins RM, Maia MdLS, Farias RHG, Camacho LAB, Freire MS, Galler R, et al. 17DD yellow fever vaccine: a double blind, randomized clinical trial of immunogenicity and safety on a dose-response study. Hum Vaccin Immunother 2013 Apr;9(4):879-888.

9. Campi-Azevedo AC, de Almeida Estevam P, CoelhoDos-Reis JG, Peruhype-Magalhaes V, Villela-Rezende G, Quaresma PF, et al. Subdoses of 17DD yellow fever vaccine elicit equivalent virological/immunological kinetics timeline. BMC Infect Dis 2014;14:391. 